\title{
Avaliação microbiológica dos leitos cirúrgicos de um hospital público oncológico da região norte do Brasil
}

\author{
Microbiological evaluation of surgical beds in a public oncology hospital in northern Brazil \\ Evaluación microbiológica de lechos quirúrgicos en un hospital oncológico público del norte de
}

Brasil

Recebido: 30/12/2020 | Revisado: 31/12/2020 | Aceito: 04/01/2021 | Publicado: 06/01/2021

\author{
Davis Wilker Nascimento Vaz \\ ORCID: https://orcid.org/0000-0002-0601-8194 \\ Universidade do Estado do Pará, Brasil \\ E-mail: daviswilkervaz@gmail.com \\ Yasmin Martins de Sousa \\ ORCID: https://orcid.org/0000-0003-3744-920X \\ Universidade do Estado do Pará, Brasil \\ E-mail: yasminmartinsdesousa@hotmail.com \\ Rosiane Luz Cavalcante \\ ORCID: https://orcid.org/0000-0002-4701-4245 \\ Universidade Federal do Pará, Brasil \\ E-mail: rosianeluzz@gmail.com \\ Maria das Graças Carvalho Almeida \\ ORCID: https://orcid.org/0000-0003-3110-1835 \\ Universidade do Estado do Pará, Brasil \\ E-mail: gravalho@gmail.com \\ Karla Valéria Batista Lima \\ ORCID: https://orcid.org/0000-0001-5807-0392 \\ Universidade do Estado do Pará, Brasil \\ E-mail: karlavaleria_2007@hotmail.com \\ Ademir Ferreira da Silva Júnior \\ ORCID: https://orcid.org/0000-0001-9121-1206 \\ Universidade Federal do Pará, Brasil \\ E-mail: ademirjunior@ufpa.br
}

\begin{abstract}
Resumo
Objetivo: avaliar as condições microbiológicas dos leitos de um Centro Cirúrgico de um Hospital público de ensino do Município de Belém, Pará. Métodos: Executou-se a coleta de amostras dos leitos cirúrgicos após a sua desocupação, por meio de rolamento de swab umedecido em solução salina esterilizada, antes e após a desinfecção dos leitos. As amostras foram processadas no Setor de Bacteriologia do Laboratório de Análises Clinica do CESUPALAC/ CESUPA. O teste de sensibilidade aos antimicrobianos foi realizado pelo método de disco difusão por meio da técnica de Kirb Bauer e o resultado expressou-se como sensível, moderadamente resistente (intermediário) e resistente. Resultados: Nas amostras coletadas dos leitos cirúrgicos, foram isoladas diferentes espécies do gênero Staphylococcus: S. aureus, S. epidermidis e S. saprophyticus, as quais apresentaram mais resistência à Penicilina, Azitromicina e Vancomicina, respectivamente. Após a desinfecção, observou-se uma redução das espécies S. aureus e S. epidermidis e um aumento da S. saprophyticus. Com relação às Gram negativas, de todas as amostras coletadas, só em duas elas positivaram, sendo ambas na coleta antes da desinfecção dos leitos. Na coleta após a desinfecção, as amostras Gram negativas negativaram. A desinfecção foi mais efetiva para as bactérias Gram negativas do que Gram positivas. Conclusão: Portanto, a análise microbiológica realizada nos leitos cirúrgicos, antes e após a desinfecção, demonstrou que eles possuíam uma carga microbiana elevada, constituindo assim, uma possível fonte de contaminação ambiental capaz de desencadear um processo infeccioso.
\end{abstract}

Palavras-chave: Infecção hospitalar; Desinfecção; Microbiologia; Leitos.

\begin{abstract}
Objective: to evaluate the microbiological conditions of the beds in a Surgical Center of a public teaching hospital in the city of Belém, Pará. Methods: Samples of the surgical beds were collected after being vacated, using a swab roll moistened in sterile saline solution, before and after bed disinfection. The samples were processed in the Bacteriology Sector of the Clinical Analysis Laboratory of CESUPA-LAC / CESUPA. The antimicrobial sensitivity test was performed using the disk diffusion method using the Kirb Bauer technique and the result was expressed as sensitive, moderately resistant (intermediate) and resistant. Results: In the samples collected from the surgical beds, different species of the Staphylococcus genus were isolated: S. aureus, S. epidermidis and S. saprophyticus, which showed
\end{abstract}


more resistance to Penicillin, Azithromycin and Vancomycin, respectively. After disinfection, there was a reduction in the species S. aureus and S. epidermidis and an increase in S. saprophyticus. Regarding Gram negative, of all samples collected, only in two they were positive, both being in the collection before disinfecting the beds. In the collection after disinfection, Gram negative samples were negative. Disinfection was more effective for Gram negative than Gram positive bacteria. Conclusion: Therefore, the microbiological analysis performed on surgical beds, before and after disinfection, demonstrated that they had a high microbial load, thus constituting a possible source of environmental contamination capable of triggering an infectious process.

Keywords: Hospital infection; Disinfection; Microbiology; Beds.

\section{Resumen}

Objetivo: evaluar las condiciones microbiológicas de las camas en un Centro Quirúrgico de un hospital público docente de la ciudad de Belém, Pará. Métodos: Se recolectaron muestras de las camas quirúrgicas luego de ser desocupadas, utilizando un rollo de hisopo humedecido en solución salina estéril, antes y después de la desinfección de la cama. Las muestras fueron procesadas en el Sector de Bacteriología del Laboratorio de Análisis Clínicos de CESUPA-LAC / CESUPA. La prueba de sensibilidad antimicrobiana se realizó mediante el método de difusión en disco mediante la técnica de Kirb Bauer y el resultado se expresó como sensible, moderadamente resistente (intermedio) y resistente. Resultados: En las muestras recolectadas de los lechos quirúrgicos se aislaron diferentes especies del género Staphylococcus: S. aureus, S. epidermidis y S. saprophyticus, que mostraron mayor resistencia a Penicilina, Azitromicina y Vancomicina, respectivamente. Después de la desinfección, hubo una reducción en las especies S. aureus y S. epidermidis y un aumento en S. saprophyticus. En cuanto a los Gram negativos, de todas las muestras recolectadas, solo en dos resultaron positivas, estando ambas en la recolección antes de desinfectar las camas. En la colección después de la desinfección, las muestras Gram negativas fueron negativas. La desinfección fue más eficaz para las bacterias Gram negativas que para las Gram positivas. Conclusión: Por tanto, el análisis microbiológico realizado en los lechos quirúrgicos, antes y después de la desinfección, demostró que tenían una alta carga microbiana, constituyendo así una posible fuente de contaminación ambiental capaz de desencadenar un proceso infeccioso.

Palabras clave: Infección hospitalaria; Desinfección; Microbiología; Camas.

\section{Introdução}

O centro cirúrgico (CC) é considerado uma área crítica do hospital que apresenta importantes desafios no que se refere ao fornecimento de um serviço seguro e de qualidade, em virtude da alta complexidade dos procedimentos realizados. Nesse contexto, são necessários cuidados especiais para evitar o surgimento das denominadas Infecções de Sítio Cirúrgico (ISC) (Fernandes et al., 2020; Santos et al., 2020).

A ISC pode ser definida como uma complicação de uma cirurgia, podendo afetar tecidos, órgãos ou cavidades. O seu diagnóstico pode ser feito entre o período de 30 dias até três meses após o procedimento cirúrgico. Na grande maioria dos hospitais, a ISC constitui-se como um dos principais tipos das Infecções Relacionadas à Assistência à Saúde (IRAS) (Souza et al., 2019).

Para prevenir as ISC é preconizado que, além do sistema de ventilação positiva, do controle do fluxo de pessoas e da paramentação adequada da equipe cirúrgica, haja, no centro cirúrgico, uma rígida rotina de assepsia e desinfecção das superfícies ambientais, como equipamentos, paredes e pisos para assim combater a colonização microbiana (Amaral et al., 2013; Moura et al., 2017).

Contudo, por se tratar de um processo mecânico, falhas durante o processo de higienização podem ocorrer. Assim, as superfícies ambientais, como os colchões cirúrgicos, podem se apresentar como um significativo reservatório de patógenos e por conseguinte, aumentar o risco do surgimento das ISC (Lourenzo et al., 2020).

As ISC atreladas aos microrganismos multirresistentes propiciam diversas implicações importantes no contexto da saúde pública, dentre elas, maior período de internação, maior tempo para o tratamento de patologias, assim como a diminuição da eficácia de drogas já conhecidas, limitando assim as possibilidades terapêuticas no controle das infecções (Gil et al., 2018; Rodrigues et al., 2020).

Diversos estudos apontam que, além de poderem ocasionar IRAS de difícil tratamento, os microrganismos multirresistentes, que podem estar presentes nas superfícies dos colchões do CC, podem também dificultar o processo de 
higienização, com o patógeno permanecendo na superfície mesmo após a sua desinfecção (Lourenzo et al., 2020).

Dado a relevância do tema e a escassez de estudos comparativos sobre as condições microbiológicas dos colchões cirúrgicos, foi proposto este estudo com o objetivo de avaliar as condições microbiológicas dos leitos de um Centro Cirúrgico de um Hospital Público de Ensino do município de Belém, no Estado do Pará.

\section{Metodologia}

Trata-se de um estudo do tipo descritivo com abordagem quantitativa e qualitativa realizado em um Centro Cirúrgico de um Hospital Público de Ensino do Município de Belém.

Executou-se a coleta de amostras de seis colchões do Centro Cirúrgico, por meio de rolamento de swab umedecido em solução salina esterilizada em $10 \mathrm{~cm} 2$ das áreas superior, média e inferior, sendo a primeira imediatamente após a desocupação dos leitos e outra após a desinfecção que é realizada pela equipe de limpeza ou de enfermagem responsável pela atividade.

Cada um destes swabs foi imediatamente imerso em tubos contendo Caldo BHI (Brain Heart Infusion), previamente identificados e, posteriormente, transportados em temperatura ambiente, protegidos em caixa térmica hermeticamente fechada, para o Setor de Bacteriologia do Laboratório de Análises Clínica do CESUPA- LAC/ CESUPA, onde foram processados. Os tubos com as amostras coletadas foram incubados em estufa bacteriológica por $24 \mathrm{~h} / 37^{\circ} \mathrm{C}$ e, após este período, semeados em ágar sal manitol, ágar sangue e ágar EMB (Eosin Methylene Blue) e incubados por $24 \mathrm{~h} / 37^{\circ} \mathrm{C}$. Os isolados bacterianos foram identificados por meio de provas bioquímicas.

$\mathrm{O}$ teste de sensibilidade aos antimicrobianos foi realizado pelo método de disco difusão por meio da técnica de Kirb Bauer, usando-se os meios de cultura Mueller Hinton (MHB, Oxoid, Basingstoke, Inglaterra) e discos com antimicrobianos individuais (Oxoid, Basingstoke, Inglaterra). A leitura foi procedida pela medição dos diâmetros dos halos de inibição e a interpretação de acordo com o padrão estabelecido pelo CLSI Standards e o resultado expressou-se como sensível, moderadamente resistente (intermediário) e resistente.

Para a análise dos dados, os resultados obtidos foram agrupados em tabelas no programa Microsoft Office Excel® 2007 e organizados em figuras com números absolutos e relativos. A pesquisa foi aprovada no Comitê de Ética e Pesquisa/ CEP do Hospital Ophir Loyola/ HOL, processo n 249555, obedecendo todos os trâmites legais da instituição.

\section{Resultados}

Foram isoladas diferentes espécies do gênero Staphylococcus: S. epidermidis, S. aureus e S. saprophyticus antes e após a desinfecção dos colchões.

Foi observado redução das unidades formadoras de colônias (UFCA) para as espécies S. aureus e S. epidermidis após a realização da desinfecção, como é demonstrado na tabela 1. Porém, quando analisados os dados do S. saprophyticus, foi visto que após a limpeza/desinfecção o número de amostras resistentes foi maior (Tabela 1). Dessa forma, pode-se inferir que, frente a análise realizada, o nível de eficácia da limpeza e da desinfecção contra S. saprophyticus é menor do que para as outras duas espécies.

A Tabela 1 também mostra os perfis de resistência aos antimicrobianos apresentados pelas três espécies Gram positivas encontradas nos colchões examinados (S. epidermidis, S. aureus e S. saprophyticus) antes e após a limpeza/desinfecção. 
Tabela 1: Perfil de resistência apresentado pelas espécies do Gênero Staphylococcus antes e após desinfecção.

\begin{tabular}{|l|l|l|l|l|l|l|}
\hline BACTÉRIA & \multicolumn{2}{|c|}{ S. aureus } & \multicolumn{2}{c|}{ S. epidermidis } & \multicolumn{2}{c|}{ S. saprophyticus } \\
\hline Antimicrobianos & Antes & Após & Antes & Após & Antes & Após \\
\hline AMC & 6 & 1 & 4 & 0 & 5 & 8 \\
\hline AZM & 7 & 1 & 7 & 5 & 15 & 15 \\
\hline DA & 6 & 0 & 5 & 3 & 11 & 14 \\
\hline CN & 3 & 0 & 1 & 1 & 5 & 7 \\
\hline C & 1 & 0 & 2 & 1 & 6 & 10 \\
\hline OFX & 2 & 0 & 1 & 1 & 5 & 7 \\
\hline OX & 4 & 2 & 5 & 2 & 7 & 10 \\
\hline LOM & 3 & 0 & 1 & 1 & 6 & 8 \\
\hline SXT & 7 & 0 & 3 & 2 & 7 & 12 \\
\hline LZD & 1 & 0 & 1 & 0 & 8 & 10 \\
\hline P & 8 & 3 & 5 & 4 & 9 & 14 \\
\hline RD & 1 & 1 & 2 & 0 & 6 & 9 \\
\hline VA & 2 & 0 & 1 & 1 & 15 & 20 \\
\hline
\end{tabular}

Legenda: amoxacilina /ac. clavulanico (AMC), azitromicina (AZM), clindamicina (DA), gentamicina $(\mathrm{CN})$, cloranfenicol $(\mathrm{C})$, ofloxacina(OFX), oxacilina (OX),

lomefloxacina (LOM), sulfametoxazol/trimetropim (STX), Linezulide (LZD),

Penicilina (P), Rifampicina (RD), Vancomicina (VA).

Fonte: Autores.

A pesquisa também revelou a incidência da resistência sobre cada espécie de Staphylococcus. Destes podemos destacar o $S$. aureus com maior resistência à Penicilina, S. epidermidis à Azitromicina e S. saprophyticus à Vancomicina, conforme a Figura 1 .

Figura 1: Perfil de resistência das espécies de Staphylococcus isoladas.

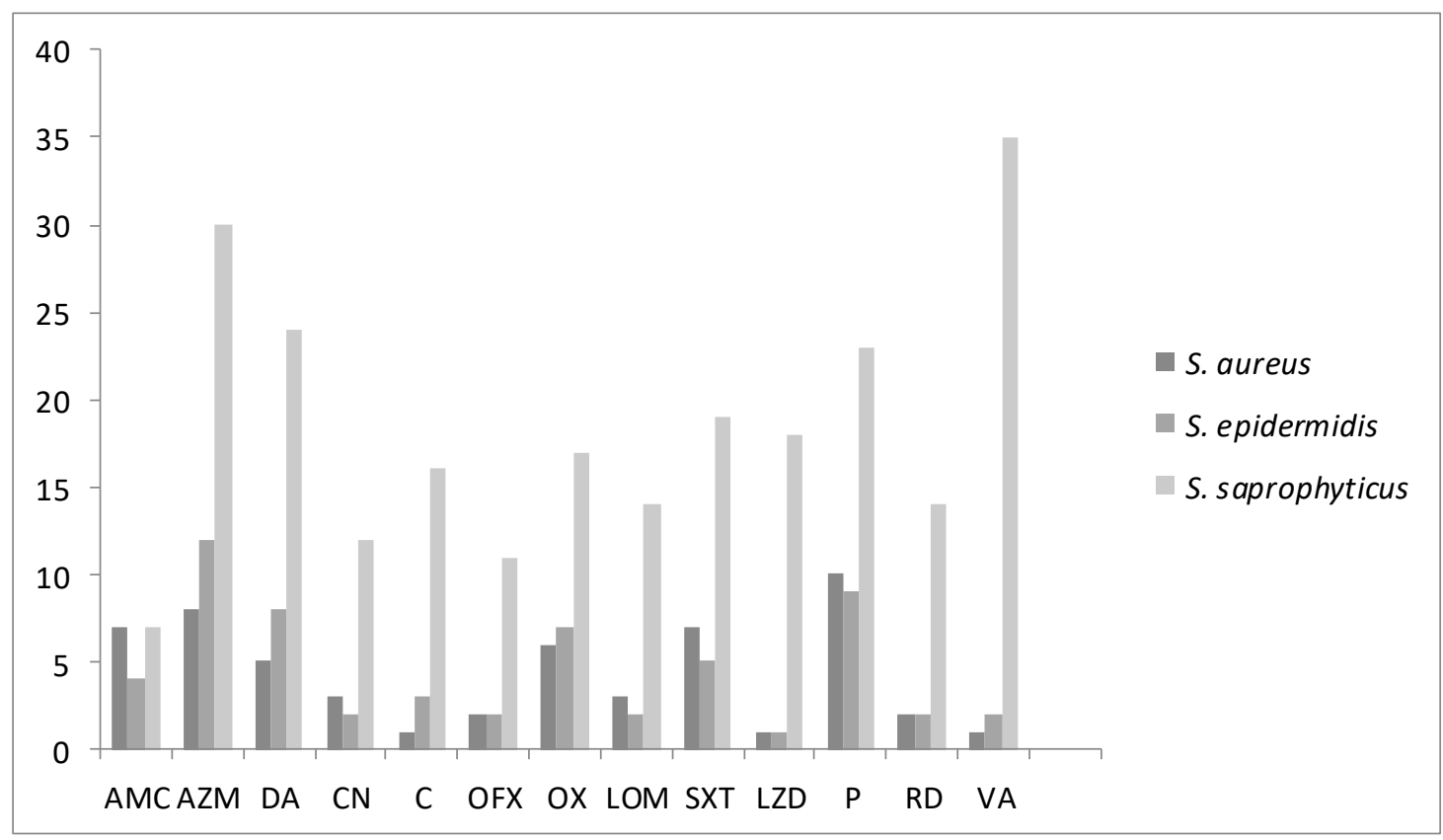

Legenda: amoxacilina /ac. clavulanico (AMC), azitromicina (AZM), clindamicina (DA), gentamicina $(\mathrm{CN})$, cloranfenicol $(\mathrm{C})$, ofloxacina $(\mathrm{OFX})$, oxacilina $(\mathrm{OX})$,

lomefloxacina (LOM), sulfametoxazol/trimetropim (STX), Linezulide (LZD),

Penicilina (P), Rifampicina (RD), Vancomicina (VA)

Fonte: Autores.

Com relação à resistência a antimicrobianos, os resultados indicam que, no geral, os patógenos foram mais resistentes à azitromicina (50 ocorrências) e à penicilina (43 ocorrências).

Quando analisadas as amostras para bactérias Gram negativas, de todas as amostras coletadas, só em duas elas 
positivaram, sendo ambas na coleta da primeira amostra dos leitos. Após a realização da limpeza/desinfecção dos respectivos colchões, as amostras negativaram. Portanto, pode-se depreender que a limpeza/desinfecção realizada nos leitos cirúrgicos foi mais efetiva contra as bactérias Gram negativas do que Gram positivas.

Com relação à resistência a antimicrobianos, a Tabela 2 indica que duas bactérias Gram negativas (K. pneumoniae e E. coli) foram resistentes a seis antimicrobianos (Cloranfenicol, Gentamicina, Ciprofloxacina, Imipinem, Ceftriaxona e Ampicilina), enquanto somente a E.coli foi resistente a dois antimicrobianos (Piperacilina e Ampicilina + Sulbactan) e um antimicrobiano (Piperacilina + Tazobactan) não apresentou resistência.

Tabela 2: Perfil de resistência da enterobacterias isoladas antes e após a limpeza/desinfecção.

\begin{tabular}{|l|l|l|l|l|}
\hline BACTÉRIA & \multicolumn{2}{l|}{ K. pneumoniae } & \multicolumn{2}{l|}{ E. coli } \\
\hline Antimicrobianos & Antes & Após & Antes & Após \\
\hline C & R & - & $\mathrm{R}$ & - \\
\hline CIP & $\mathrm{R}$ & - & $\mathrm{R}$ & - \\
\hline CN & $\mathrm{R}$ & - & $\mathrm{R}$ & - \\
\hline PIP/TAZO & $\mathrm{S}$ & - & $\mathrm{S}$ & - \\
\hline IPM & $\mathrm{R}$ & - & $\mathrm{R}$ & - \\
\hline PRL & $\mathrm{S}$ & - & $\mathrm{R}$ & - \\
\hline CRO & $\mathrm{R}$ & - & $\mathrm{R}$ & - \\
\hline ASB & $\mathrm{S}$ & - & $\mathrm{R}$ & - \\
\hline AMP & $\mathrm{R}$ & - & $\mathrm{R}$ & - \\
\hline
\end{tabular}

Legenda: cloranfenicol (C), ciprofloxacina (CIP), gentamicina $(\mathrm{CN})$, piperacilina + tazobactan (PIP/TAZO),imipinem (IPM), piperacilina (PRL), ceftriaxona (CRO), ampicilina + sulbactan (ASB), ampicilina (AMP).

Fonte: Autores.

\section{Discussão}

A análise microbiológica dos colchões cirúrgicos deve se constituir como prática de avaliação da qualidade dos serviços, detectando inconformidades e possibilitando a correção de processos a fim de minimizar a ocorrência de infecção hospitalar (Verli, 2019; Frota et al., 2017).

No presente estudo foi constatado que a bactéria do gênero Staphylococcus foi a única reagente Gram positiva nas amostras coletadas dos colchões/mesas cirúrgicas, variando entre as espécies S. epidermidis, S. aureus e S. saprophyticus.

Já foram contabilizadas mais de 30 espécies de Staphylococcus. Habitualmente, esse gênero faz parte da microbiota normal das mucosas do trato respiratório, urogenital e digestivo de seres humanos e animais, porém caso as barreiras naturais, estejam comprometidas por cirurgia ou trauma, algumas dessas espécies podem ocasionar infecções de caráter oportunista (Silva et al., 2018).

Os Staphylococcus possuem como mais importante reservatório o ser humano, sendo comum à infecção cruzada, por meio das vias aéreas ou do contato direto com pessoas e objetos contaminados pelas mãos dos funcionários da unidade. Ademais, as bactérias do gênero Staphylococcus podem sobreviver em superfícies secas durante longos períodos, o que favorece a sua permanência no ambiente nosocomial (Lavor et al., 2019; Rodrigues et al., 2019).

A espécie de Staphylococcus de maior interesse médico, principalmente em ambiente hospitalar, é o S. aureus que tem se mostrado como um dos patógenos com grande prevalência em infecções nosocomiais. Isto condiz com os resultados do presente estudo visto que, das 72 coletas realizadas, 13 (18\%) pertenciam ao S. aureus (Ramírez, 2019; Oliveira et al., 2019). 
A colonização pelo S. aureus comumente é desprovida de sintomas, assim, o indivíduo pode se tornar carreador do microrganismo mesmo apresentando-se assintomático. Logo, os próprios profissionais de saúde podem atuar como carreadores assintomáticos de S. aureus e, por conseguinte, disseminar o microrganismo para os indivíduos hospitalizados (Silva et al., 2019; Lopes et al., 2018; Pereira et al., 2019).

Até a década de 60, o tratamento antimicrobiano para infecções por staphylococcus aureus era realizado sem complicações com o uso da penicilina. Entretanto, ao mesmo tempo em que o uso desse antimicrobiano tornava-se cada vez mais difundido para o tratamento de doenças infecciosas, evidências de resistência bacteriana a ele começaram a ser descobertas. Com o objetivo de tentar resolver o problema, a meticilina, um betalactâmico sintético resistente a ação das betalactamases que o S. aureus produz, foi introduzido. No entanto, pouco tempo depois, começou a surgir amostras resistentes a esse novo antimicrobiano. Essas cepas, chamadas de Staphylococcus aureus resistente a meticilina (MRSA), caracterizam-se por apresentar resistência a todos os antibióticos ß-lactâmicos (Harkins et al., 2017; Rodrigues et al., 2019).

O presente estudo também identificou um caso resistente à Vancomicina. Esse antibiótico era considerado uma droga revolucionária quando surgiu, justamente por apresentar eficiência contra as cepas MRSA. Porém, eventualmente, a eficácia dessa droga iria ter fim, pois a maioria a utilizava de maneira abusiva, contribuindo assim para que ocorra a indução ou seleção de cepas de bactérias tolerantes, levando a ineficácia do tratamento e resistência ao fármaco. No começo dos anos 2000 , algumas cepas de Staphylococcus Aureus resistente à vancomicina (SARV) foram isoladas. Em 2012, notificou-se isolados SARV nos Estados Unidos, Índia e Irã. No Brasil, no ano de 2013, publica-se o primeiro caso de SARV (Lázaro et al., 2019).

Com relação às bactérias Gram negativas, a pesquisa revelou que dentre as amostras coletadas estavam a Klebsiella pneumoniae e a Escherichia Coli, sendo cada uma reagente uma vez. Os dois microrganismos foram negativados após a realização da limpeza/desinfecção.

A espécie Klebsiella Pneumoniae é um bacilo gram-negativo presente no trato gastrointestinal de indivíduos colonizados, que pode sobreviver por muito tempo na pele e em ambientes secos, como superfícies hospitalares. É importante causa de infecções relacionadas à assistência à saúde, tanto no meio ambiente comunitário quanto no hospitalar. O número de surtos hospitalares causados por K. pneumoniae é cada vez maior com a mudança no padrão de sensibilidade aos antimicrobianos (Siva et al., 2019).

Moura et al. (2007), visando determinar a prevalência de IH e distribuição por topografia e por microrganismo e suas sensibilidades antimicrobianas, determinou que dos 394 casos analisados em seu estudo, 132 (33,67\%) foram pelo agente K. pneumoniae, que se mostrou resistente ao antimicrobiano Imipinem em 53 amostras.

No presente estudo, o microrganismo também se mostrou resistente ao Imipinem e à outras drogas como Cloranfenicol, Ciprofloxacino, Gentamicina, Ampicilina e Ceftrizaxona, sendo sensível somente a Piperacilina + Tazobactan e Ampicilina + Sulbactan.

No que se diz respeito ao outro microrganismo gram negativo encontrado, a E. coli, embora faça parte da microbiana normal do intestino humano, ele é responsável por cerca de $80 \%$ das infecções urinárias. Assim como a Klebisiella, também produz a Betalactamase, sendo também considerado um grande problema de saúde pública (Costa et al., 2019).

Na presente pesquisa a E.coli se mostrou resistente a todos os antimicrobianos testados (Ciprofloxacina, Ceftriaxona, Gentamincina, Imipinem, Piperacilina, Ampicilina + Sulbactan e Cloranfenicol), com exceção da Piperacilina + Tazobactan. Garcia et al. (2013), buscando definir o perfil epidemiológico das infecções hospitalares causadas por bactérias multirresistentes em um hospital do norte de Minas Gerais, identificou que dos 44 casos de IH por bactérias multirresistentes, $11(23,4 \%)$ foram por E.coli, resistente a Gentamicina, Ciprofloxacina, Ceftazidima e Ceftriaxona. 


\section{Conclusão}

Nas amostras coletadas foram identificadas bactérias Gram Positivas e negativas, sendo que dentre as Gram positivas estava o gênero Staphylococcus, variando entre as espécies S. epidermidis, S.aureus e S. saprophyticus. Já entre as Gram negativas, foram identificadas a Escherichia coli e a Klebsiellapneumoniae.

As bactérias da espécie S. epidermidis se mostraram mais resistentes para Azitromicina, S. aureus mais resistente para Penicilina e S. saprophyticus mais resistentes para Vancomicina. Já entre as Gram negativas, a E. coli se mostrou resistente aos seguintes antimicrobianos testados: Ciprofloxacina, Ceftriaxona, Gentamicina, Imipinem, Piperacilina, Ampicilia + Sulbactan e Cloranfenicol. Já a K. pneumoniae demonstrou resistência ao Imipinem e à outras drogas como Cloranfenicol, Ciprofloxacina, Gentaminicina, Ampicilina e Ceftriaxona.

Portanto, a análise microbiológica realizada nos leitos cirúrgicos, antes e após a desinfecção, demonstrou que eles possuíam uma carga microbiana elevada, constituindo assim, uma possível fonte de contaminação ambiental capaz de desencadear um processo infeccioso.

Por fim, é válido ressaltar que as pesquisas que envolvem a análise microbiológica de leitos cirúrgicos de Centro Cirúrgico antes e após a desinfecção ainda são muito escassos, logo, faz-se necessário a condução de novos estudos que avaliem a eficácia da limpeza dos leitos e das demais superfícies hospitalares em outros locais, com o intuito melhorar essa prática e trazer benefícios em prol da segurança do paciente.

\section{Referências}

Amaral, A. M., Filho, A. D., Sousa, M. M. A., Barbosa, P. A., \& Filho, P. P. G. (2013). Importância da proteção da mesa de instrumentais cirúrgicos na contaminação intraoperatória de cirurgias limpas. Revista Latino-Americana de Enfermagem, 21(1).

Costa, M. C. P., Lima, K. M., Dellacqua, M. N., \& Santos, V. E. F. A. (2019). Micro-Organismos Isolados A Partir De Espécimes Clínicos De Centro Cirúrgico. Revista Saúde e Desenvolvimento, 13(14).

Fernandes, L. F. G., Fassarella, C. S., Cavalcanti, R. S., Camerini, F. G., Meneses, R. O., \& Souza, R. M. (2020). Cultura de segurança em centro cirúrgico universitário. Research, Society and Development, 9(8), e119985164.

Frota, O. P., Ferreira, A. M., Guerra, O. G., Rigotti, M. A., Andrade, D., Borges, N. M. A., \& Almeida, M. T. G. (2017). Eficiência da limpeza e desinfecção de superfícies: correlação entre métodos de avaliação. Revista Brasileira de Enfermagem, 70(6), 1242-1249.

Garcia, L. M., César, I. C. O., Braga, C. A., Souza, G. A. A. D., \& Mota, E. C. (2013). Perfil epidemiológico das infecções hospitalares por bactérias multidrogarresistentes em um hospital do norte de Minas Gerais. Revista de Epidemiologia e Controle de Infecção, 3(2), 45-49.

Gil, A. C., Bordignon, A. P. P., Castro, E. A. R., Castro, S. T., Rafael, R. M. R., \& Pereira, J. A. A. (2018). Avaliação microbiológica de superfícies em terapia intensiva: reflexões sobre as estratégias preventivas de infecções nosocomiais. Revista Enfermagem Uerj, 26:e26388.

Harkins, C. P., Pichon, B., Doumith, M., Parkhill, J., Westh, H., Lencastre, A. T. H., Bentley, S. D., Kearns, A. M., \& Holden, M. T. G. (2017). Methicillinresistant Staphylococcus aureus emerged long before the introduction of methicillin into clinical practice. Genome Biology, 18(130).

Lavor, M. L. S. S., Neto, A. A. A., Souza, R. C., Silva, H. C. L., Freitas, R. B., \& Pereira, M. S. V. (2019). Colonização microbiana por staphylococcus aureus multirresistentes em aparelho celular. Temas em Saúde, 19(2), 234-241.

Lázaro, L. F., Vela, A., Leonardo, A. (2019). Staphylococcus aureus y el perfil de resistencias desde la penicilina hasta la vancomicina. Rev UNIANDES Ciencias de la Salud, 2(3), 230-239.

Lopes, L. P., Pio, D. P. M., Pereira, F. M. V., Menegueti, M. G., Freitas, J. P. \& Gir, E. (2018). Prevalência de Staphylococcus aureus resistente à oxacilina isolado em profissionais de enfermagem. Ver Rene (Online), 19:e32478.

Lourenzo, M. A. R., Lima, K. C., Almeida, N. B., \& Aguiar, A. A. (2020). Contaminação em superfícies de uti após limpeza/desinfecção no brasil: uma revisão integrativa. Revista de Patologia do Tocantins, 10(4), 27-32.

Moura, L. C. D., Carvalho, L. N., Silva, R. S., \& Gouveia, B. L. A. (2017). Higiene e desinfecção hospitalar aliadas na segurança do paciente. Temas em saúde, 17(1), 4-17.

Moura, M. E. B., Campelo, S. M. A., Brito, F. C. P., Batista, O. M. A., Araújo, T. M. E., \& Oliveira, A. D. S. (2007). Infecção hospitalar: estudo de prevalência em um hospital público de ensino. Revista Brasileira de Enfermagem, 60(4), 416-421.

Oliveira, W. V., Santos, W. S., Gomes, B. S., \& Lima, J. L. C. (2019). Etiologia e perfil de susceptibilidade dos microrganismos isolados de hemoculturas no Hospital das Clínicas da UFPE no período de janeiro a dezembro de 2014. Revista Brasileira de Análises Clínicas, 51(1), 40-45. 
Research, Society and Development, v. 10, n. 1, e16610111602, 2021

(CC BY 4.0) | ISSN 2525-3409 | DOI: http://dx.doi.org/10.33448/rsd-v10i1.11602

Pereira, B. L., Santos, A. C. D., Aquino, J. M., Neto, G. R. A., Souza, A. A. D., Rios, B. R. M., Leão, C. D. A., Pereira, S. G. S., Siqueira, L. G., \& Piris, Á. P. (2019). Colonização por microrganismos em colaboradores da saúde. Enfermagem Brasil, 18(2): 213-219.

Ramírez, E. M. (2019). Caracterización de la bacteremia por Staphylococcus aureus en pacientes hospitalizados del Hospital San Juan de Dios entre enero 2015 a diciembre 2017. Revista Medicina Legal de Costa Rica, 36(1).

Rodrigues, A. W. S., Camargo, B., \& Maciel, E. (2019). Pesquisa de staphylococcus aureus resistente a meticilina (mrsa) em elevadores de um hospital da rede privada de brasília - DF. Revista Brasileira de Pesquisa em Ciências da Saúde, 6(11), 13-18.

Rodrigues, I. P., Turnes, O., \& Castro, A. F. (2020). Contribuição do gráfico de controle de somas acumuladas na assistência e segurança do paciente com base no monitoramento da incidência de bactérias multirresistentes em uma unidade de terapia intensiva. Brazilian Journal of health Review, 3(4), 10543-10558.

Santos, R. M., Joaquim, F. L., Souza, D. F., \& Souza, C. J. (2020). Infecção do sítio cirúrgico em cirurgias cardíacas: fatores que influenciam na prevenção e controle da infecção e as atribuições da Enfermagem; uma revisão integrativa. Research, Society and Development, 9(8), e71985213.

Silva, G. B., Neto, M. L., Texeira, A. N., Valle, A. M., \& Alves, F. A. (2019). Estudo da prevalencia de staphylococcus aureus resistentes colonizando estudantes da faculdade de medicina em campos dos goytacazes. Revista Interdisciplinar do Pensamento Científico, 2(5), 252-262.

Silva, J. G., Alcântara, A. M., \& Mota, R. A. (2018). Mastite bovina causada por Staphylococcus spp. resistentes à meticilina: revisão de literatura. Pesquisa Veterinária Brasileira, 38(2), 223-228.

Siva, E. L., Silva, M. S., Aoyama, E. A., \& Souza, R. A. G. (2019). Klebsiella pneumoniae carbapenamase (kpc): bactéria multirresistente a antibióticos. Revista Brasileira Interdisciplinar de Saúde, 1(1), 62-66.

Souza, V. P. R., Tinoco, V. A., \& Carmo, G. T. (2019). Atuação do Enfermeiro no Processo de Cirurgia Segura. Revista Transformar, 13(1), 540-559.

Verli, M. V. A., \& Gonçalves, L. C. O. (2019). Uma visão ampla das infecções hospitalares. Revista Panorâmica, 27, 178-194. 